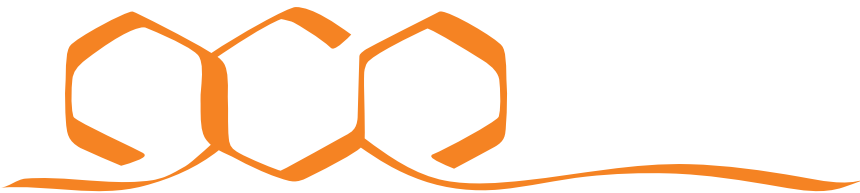 \\ COMMUNICATIONS \\ CHEMISTRY
}

ARTICLE

https://doi.org/10.1038/s42004-019-0114-7

OPEN

\section{Direct atomic identification of cation migration induced gradual cubic-to-hexagonal phase transition in $\mathrm{Ge}_{2} \mathrm{Sb}_{2} \mathrm{Te}_{5}$}

Yonghui Zheng ${ }^{1}$, Yong Wang ${ }^{1}$, Tianjiao Xin ${ }^{1}$, Yan Cheng ${ }^{1,2}$, Rong Huang ${ }^{2}$, Pan Liu ${ }^{3}$, Min Luo ${ }^{4}$, Zaoli Zhang ${ }^{5}$, Shilong Lv' ${ }^{1}$, Zhitang Song ${ }^{1} \&$ Songlin Feng ${ }^{1}$

$\mathrm{GeTe}_{\mathrm{Sb}} \mathrm{Te}_{3}$ pseudobinary system, especially $\mathrm{Ge}_{2} \mathrm{Sb}_{2} \mathrm{Te}_{5}$ alloy, is the most desirable material to be commercialized in phase change random access memory. Directly resolving the local atomic arrangement of $\mathrm{Ge}_{2} \mathrm{Sb}_{2} \mathrm{Te}_{5}$ during intermediate steps is an effective method to understand its transition mechanism from face-centered-cubic to hexagonal phases. In this study, we provide insights into the atomic arrangement variation during face-centered-cubic to hexagonal transition process in $\mathrm{Ge}_{2} \mathrm{Sb}_{2} \mathrm{Te}_{5}$ alloy by using advanced atomic resolution energy dispersive $\mathrm{X}$-ray spectroscopy. Induced by thermal annealing, randomly distributed germanium and antimony atoms would migrate to the specific (111) layer in different behaviors, and antimony atoms migrate earlier than germanium atoms during the phase transition process, gradually forming intermediate structures similar to hexagonal lattice. With the migration completed, the obtained stable hexagonal structure has a partially ordered stacking sequence described as below: $-\mathrm{Te}-\mathrm{Sb}_{\mathrm{x}} / \mathrm{Ge}_{\mathrm{y}}-\mathrm{Te}-\mathrm{Ge}_{\mathrm{x}} / \mathrm{Sb}_{\mathrm{y}}-\mathrm{Te}-\mathrm{Ge}_{\mathrm{x}} / \mathrm{Sb}_{\mathrm{y}}-\mathrm{Te}-\mathrm{Sb}_{\mathrm{x}} / \mathrm{Ge}_{\mathrm{y}}-\mathrm{Te}-(\mathrm{x}>\mathrm{y})$, which is directly related to the migration process. The current visual fragments suggest a gradual transition mechanism, and guide the performance optimization of $\mathrm{Ge}_{2} \mathrm{Sb}_{2} \mathrm{Te}_{5}$ alloy.

\footnotetext{
${ }^{1}$ State Key Laboratory of Functional Materials for Informatics, Shanghai Institute of Microsystem and Information Technology, Chinese Academy of Sciences, Shanghai 200050, China. ${ }^{2}$ Key Laboratory of Polar Materials and Devices (MOE), Department of Optoelectronics, East China Normal University, Shanghai 200241, China. ${ }^{3}$ State Key Laboratory of Metal Matrix Composites, School of Materials Science and Engineering, Shanghai Jiao Tong University, Shanghai 200030, China. ${ }^{4}$ Department of Physics, Shanghai Second Polytechnic University, Shanghai 201209, China. ${ }^{5}$ Erich Schmid Institute of Materials Science, Austrian Academy of Science, Leoben 8700, Austria. Correspondence and requests for materials should be addressed to,

Y.C. (email: ycheng@ee.ecnu.edu.cn) or to Z.S. (email: ztsong@mail.sim.ac.cn)
} 
A significant advancement in the semiconductor technology has led to the use of phase change memory (PCRAM) in a commercial product to perfect the storage system ${ }^{1-3}$. As the most important key material of PCRAM, $\mathrm{Ge}_{2} \mathrm{Sb}_{2} \mathrm{Te}_{5}$ (GST) alloy ${ }^{4,5}$, is proved to have long cyclability ${ }^{6}$, high density ${ }^{7}$, and nanosecond switching speed ${ }^{8}$ characteristics. Induced by thermal annealing, amorphous (a-) GST material will crystallize into a metastable face-centered-cubic (f-) structure $(F m \overline{3} m, a=6.02 \AA)$ at $\sim 150^{\circ} \mathrm{C}$ with a cationic $\mathrm{Ge} / \mathrm{Sb} / \mathrm{vac}$-sublattice and an anionic Te f-sublattice, accompanied by more than 4 orders of magnitude resistance decreasing ${ }^{9,10}$. Such a huge resistance contrast between a- and f-phase is utilized to realize information storage in GST based PCRAM devices ${ }^{11,12}$. If f-phase material was overheated, for example, $320-350^{\circ} \mathrm{C}$, it will transform into stable trigonal structure $(P \overline{3} m 1, a-b-c$ stacking sequence feature of (0001) planes) with a further resistance decreasing, which was often indexed with hexagonal (h-) phase $(a=4.20 \AA, c=16.96 \AA)^{10,13}$.

Such a stable h-phase would worsen the storage performance due to the large reset current ${ }^{14}$, big volume shrinkage ${ }^{15}$, supernormal mechanical stress ${ }^{16,17}$, and bad cyclability ${ }^{18}$. The specific local atomic arrangements in h-phase may be also an advantage in recently proposed interfacial phase change memory (iPCM) devices $^{19}$, however in traditional T-cell structure ${ }^{3}$, phase change material is deposited without precision crystallographic texture control, and poor-quality interface with multiple grains in hphase requires substantially higher SET/RESET switching energy. Thus, preventing the formation of $\mathrm{h}$-phase is still a critical issue for PCRAM application. Unfortunately, at the moment, the atomic motif of different crystalline structures in GST alloy has not even been unambiguously resolved. As for f-phase, Yamada et al. claimed that $\mathrm{Te}$ atoms occupy the anion sites, and $\mathrm{Ge}, \mathrm{Sb}$, and intrinsic vacancies randomly occupy the cation sites ${ }^{20,21}$, resulting in an atomic stacking sequence along [111] direction of f-lattice ([0001] direction in h-lattice) depicted as below: (Te-Ge/Sb/Vacancy)-. While Sun et al. claimed that the stable configuration should make $\mathrm{Ge}, \mathrm{Sb}$ atoms, and intrinsic vacancies locate in the separate layers ${ }^{22}$, and recently they calculated that $\mathrm{f}$ phase is actually polymorphic, varying from randomly distributed vacancies to highly ordered vacancy layers ${ }^{23}$. By using an element-resolved atomic imaging method, Zhang et al. confirmed a randomly distributed vacancies $\mathrm{f}$-phase ${ }^{24,25}$, while no direct element-resolved evidence was reported for the polymorphic state in f-phase at present. As regards to stable h-phase, it is characteristic of nine-layer stacking blocks along [0001] orientation, due to the immature of the analysis means, the exact atomic arrangement is still under debate, mainly including three possible stacking sequences along [0001] orientation: (a) Kooi model $^{26}$ : $\quad-(\mathrm{Te}-\mathrm{Sb}-\mathrm{Te}-\mathrm{Ge}-\mathrm{Te}-\mathrm{Ge}-\mathrm{Te}-\mathrm{Sb}-\mathrm{Te})-$; $\quad$ (b) Petrov model $^{27}$ : $-(\mathrm{Te}-\mathrm{e}-\mathrm{Te}-\mathrm{Sb}-\mathrm{Te}-\mathrm{Sb}-\mathrm{Te}-\mathrm{Ge}-\mathrm{Te})-$; and (c) Matsunaga model ${ }^{28}:-\left(\mathrm{Te}-\mathrm{Ge}_{0.44} \mathrm{Sb}_{0.56}-\mathrm{Te}-\mathrm{Ge}_{0.56} \mathrm{Sb}_{0.44}-\mathrm{Te}-\mathrm{Ge}_{0.56} \mathrm{Sb}_{0.44}-\mathrm{Te}-\right.$ $\mathrm{Ge}_{0.44} \mathrm{Sb}_{0.56}-\mathrm{Te}$ )-. Density functional theory calculations demonstrated that the most stable configuration is favor to Kooi sequence ${ }^{22,29-31}$. While Da Silva et al. claimed that the stable configuration should make $\mathrm{Ge}$ and $\mathrm{Sb}$ atoms on the opposite side surrounded with $\mathrm{Te}$ atoms to release strain energy, and leads to the intermixing $\mathrm{Ge}$ and $\mathrm{Sb}$ atoms in the (0001) planes $^{32}$. Controversy not only appears in the theoretical aspect, but also in the experimental observations. In the absorption coefficient experiment, it claims that the Petrov sequence fits better to the measurement ${ }^{33}$. However, the large broadening phenomenon observed in Raman peaks ${ }^{34}$, and the non-uniform contrast of cationic layers ${ }^{35,36}$ from high-angle annular dark-field scanning transmission electron microscopy (HAADF-STEM) image are both inclined to Matsunaga sequence.

Considering the plausible similar atomic sequence in $\mathrm{f}-$ and $\mathrm{h}$ lattice, researchers tend to believe a diffusionless controlled mechanism should happen during the whole crystal transition process $^{22,37}$. For example, the shearing martensitic mechanism with a twisting atomic movement ${ }^{38,39}$, the epitaxial growth mechanism through a continuous accumulation ${ }^{40,41}$, and recently, a vacancy ordering mechanism which emphasizes the critical role of the vacancy aggregation behavior ${ }^{24,25,42,43}$. Though above-mentioned mechanisms seem to be very intriguing, puzzles in the atomic arrangement of different structures, especially the intermediate (i-) state, obstruct the clarification of the exact transition mechanism. Hence, tracing the atom migration behavior during the whole process would be a crucial and effective method to deduce the transition mechanism.

Here, we show how initial f-phase transforms into stable hphase at the atomic scale occurs using atomic resolution energy dispersive X-ray spectroscopy (EDS). More specifically, from initial f-phase to different degree i-phase and to h-phase, cationic atoms will gradually migrate to the specific (111) layers from the completely disordered state to partial ordered distribution. Besides, $\mathrm{Ge}$ and $\mathrm{Sb}$ atoms show different migration behaviors, resulting in different cationic ordered extent i-phases. In stable hphase, $\mathrm{Sb}$ atoms aggregate in the outer cationic layers, while Ge atoms aggregate in the inner cationic layers in the nine-layer blocks, manifesting the following suggested stacking sequence: $-\mathrm{Te}-\mathrm{Sb}_{x} / \mathrm{Ge}_{y}-\mathrm{Te}-\mathrm{Ge}_{x} / \mathrm{Sb}_{y}-\mathrm{Te}-\mathrm{Ge}_{x} / \mathrm{Sb}_{y}-\mathrm{Te}-\mathrm{Sb}_{x} / \mathrm{Ge}_{y}-\mathrm{Te}-\quad(x>y)$. Through the visual atomic identification of $\mathrm{f}$-to-h transition fragments in GST material, we claim that it is a gradual change process dominated by the directed migration of $\mathrm{Ge} / \mathrm{Sb}$ atoms, which provides a profound understanding of phase change mechanism between similar crystal structures, and guides the optimization of GST alloy for PCRAM application in the future.

\section{Results}

The direct visualization of f-phase arrangement on atomic level. Figure 1 signifies the atomic arrangement of GST alloy in metastable f-phase annealed at $150^{\circ} \mathrm{C}$ for $5 \mathrm{~min}$. Owing to the incoherent scattering intrinsic of STEM-HAADF technique, the intensity of the HAADF image is roughly proportional to $Z^{2}$ (ref. ${ }^{44}$ ), where $Z$ is the atomic number. In GST alloy, the atomic number of $\mathrm{Ge}, \mathrm{Sb}$, and $\mathrm{Te}$ is 32,51 , and 52 , respectively, and the corresponding HAADF image projected along [110] orientation demonstrates a periodical contrast-intensity fluctuation in Fig. 1a, fitting well with the assumption that $\mathrm{Ge}$ and $\mathrm{Sb}$ atoms occupy cationic sites, and Te atoms occupy anionic sites ${ }^{24,25,37,45}$. After all, HAADF method is an indirect technology to analyze the atomic arrangement of the structure, we then used atomic EDS mapping technique ${ }^{46,47}$ to directly determine the location of different elements as depicted in Fig. 1b-d, which also shows a periodical contrast-intensity fluctuation for Ge (red), Sb (green), and $\mathrm{Te}$ (blue) elements taken from the yellow rectangle area. The associated EDS profile along [111] orientation was also inset in the right side of each image, which is integrated in its perpendicular direction, the $[11 \overline{2}]$ orientation for $\mathrm{f}$-phase. By evaluating the intensity of the profile, we could qualitatively evaluate the concentration of each element, and the almost same height level at each cationic layer unambiguously demonstrates the random distribution of three elements in initial f-phase. If we overlap the atomic mapping pictures of $\mathrm{Ge}, \mathrm{Sb}$, and Te elements as shown in Fig. 1e-h, it can be clearly seen that Te atoms occupy anionic sublattice, while Ge and Sb atoms occupy cationic sublattice with plenty of vacancy sites together considering the stoichiometric of the film, in line with the Yamada atomic model ${ }^{20}$ as inset in Fig. 1a.

Ordered cation migration during f-to-h transition process. Though literatures emphasize the diffusionless mechanism during 

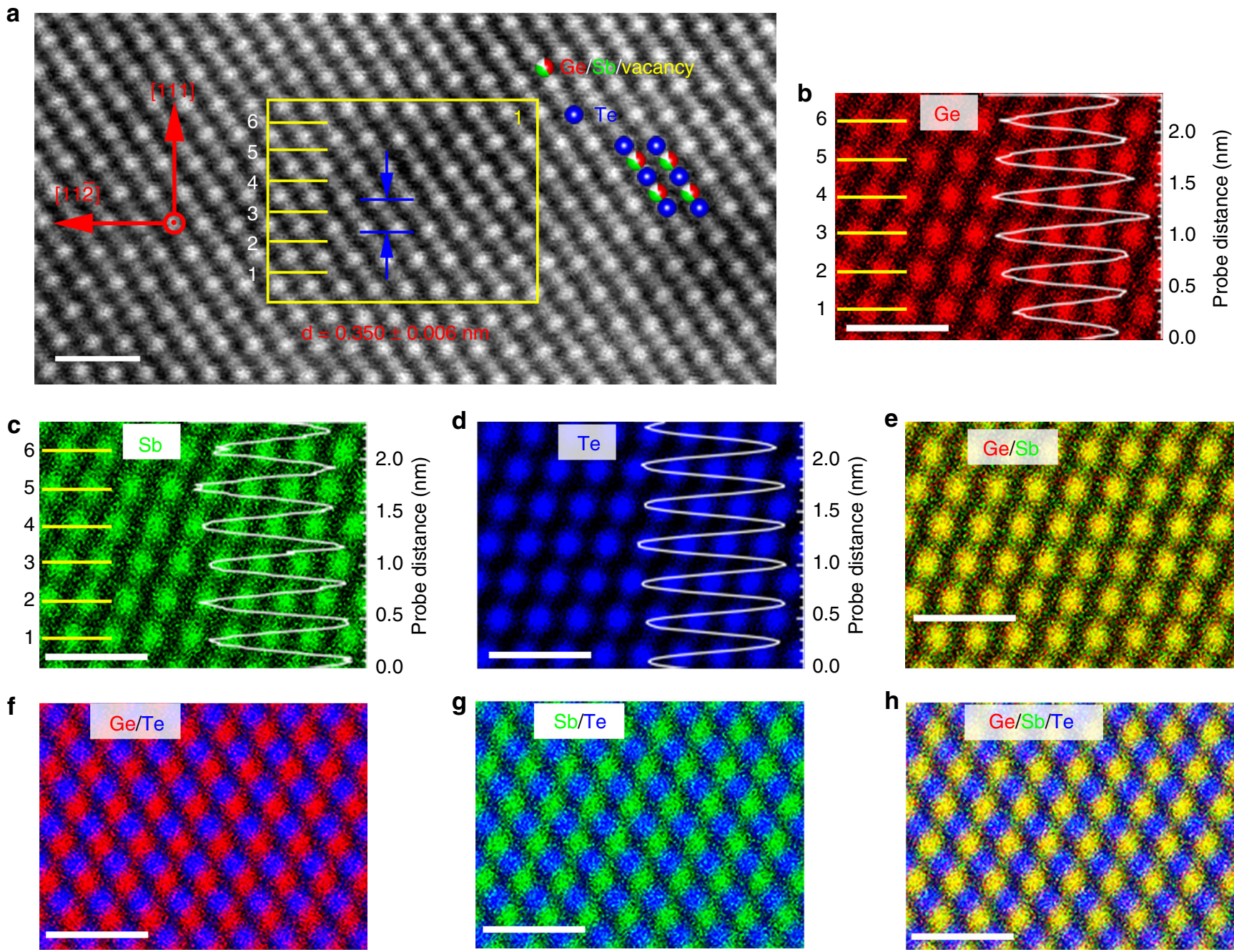

Fig. 1 Atomic resolution elemental maps of $f$-phase GST. a A prototype HAADF image of $f$-phase GST alloy projected along [110] orientation, exhibiting a periodical contrast-intensity fluctuation. The inset is the corresponding atomic model, and the distance between Te-Te layers is $0.350 \pm 0.006 \mathrm{~nm}$. Lines 1-6 denote the cationic layer, respectively. b-d Atomic resolution maps of Ge, Sb, and Te atoms taken from area 1, respectively. The EDS profiles along [111] orientation are presented on the right-hand side showing uniform height peaks. e-h The corresponding overlap results of every two elements by pairs, and the overlap of three elements, indicating that Ge/Sb atoms indeed occupy cationic sites, and Te atoms occupy anionic sites in f-phase. Scale bar: (a-h) 1 $\mathrm{nm}$

the structure transition, to date, no direct evidence were given on atom arrangements in i-phase. In the following section, the atomic arrangement of different i-phases is discussed in detail to shed light on the transition mechanism. Figure $2 \mathrm{a}$ is the HAADF image of GST film annealed at $290{ }^{\circ} \mathrm{C}$ for $5 \mathrm{~min}$ projected along [110] orientation, in which cation migration has already proceeded. The inset atomic model shown in Fig. 2a indicates the position of cation and anion layers with typical f-phase stacking sequence. Compared to Fig. 1a, one of the most prominent changes is that two horizontal dark contrast atomic columns appear as denoted by arrows (lines 1 and 6), which are separated by nine-layer blocks. Extracted from the dark atomic columns (yellow rectangular region), the corresponding contrast profile along [111] orientation has a tendency to decrease from left to right side as shown in Fig. 2b. Area " $A$ " shows uniform cation distribution almost same with Fig. 1. While the cationic layer atoms at areas "B" and "C" are partially missing, or move somewhere else. Besides, the atomic spacing of Te-Te layers above and below the dark atomic column also shows a decreasing trend, from $0.356 \pm 0.008 \mathrm{~nm}$ (area "A") to $0.346 \pm 0.010 \mathrm{~nm}$ (area "B") to $0.329 \pm 0.010 \mathrm{~nm}$ (area " $\mathrm{C}$ ") as shown in the bottom of
Fig. $2 b$, which may imply gradual migration of cationic atoms in the corresponding sites from " $\mathrm{A}$ " to " $\mathrm{B}$ " to " $\mathrm{C}$ ".

To verify it, atomic EDS mapping was utilized to explore the distribution of $\mathrm{Ge}, \mathrm{Sb}$, and Te elements in area 2 (denoted as magenta rectangle) shown in Fig. 2c-e and area 3 (denoted as orange rectangle) shown in Fig. $2 \mathrm{f}-\mathrm{h}$. The corresponding overlaps of three elements in areas 2 and 3 confirm that $\mathrm{Ge}$ and $\mathrm{Sb}$ columns occupy the cationic sites, while Te columns occupy the anionic sites in Supplementary Figure 4 and Supplementary Figure 5. When the cation starts to migrate, Sb columns no longer retain a disordered distribution in cationic site, and the corresponding EDS profile clearly shows that lines 1 and 6 possess lower peaks than other lines. As a contrast for Ge, it still shows a uniform contrast in every cationic site, and the corresponding EDS profiles projected along [111] orientation have the same level from lines 1 to 6 . Therefore, the observed dark contrast in area 2 would be firstly ascribed to the migration of $\mathrm{Sb}$ atoms.

When moving to area 3, with a further decreased concentration of cationic layer atoms in yellow rectangular region of Fig. 2a, the migrated cation is still mainly dominated by $\mathrm{Sb}$, showing a deeper 

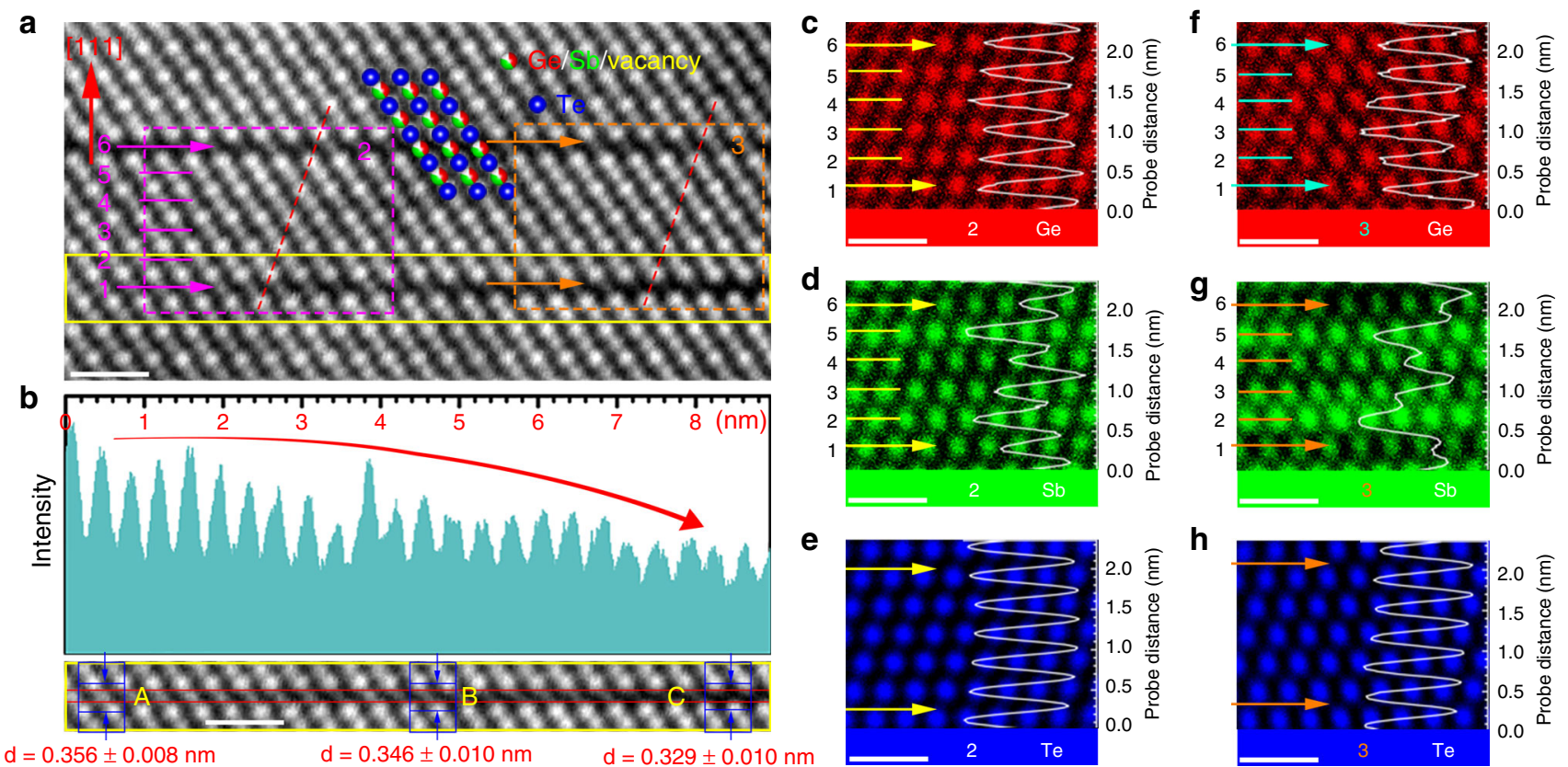

e

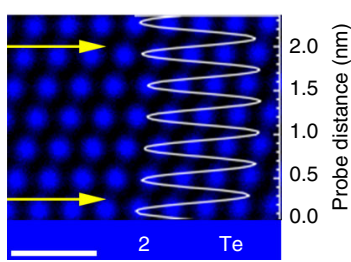

h

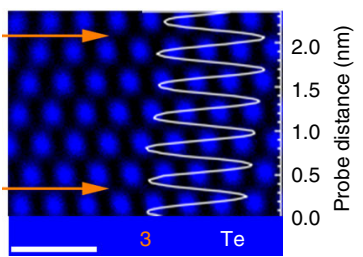

Fig. 2 Atomic resolution elemental map of i-phase GST at initial stage. a The HAADF image of i-phase GST alloy projected along [110] orientation, the dashed white line along anionic atoms shows f-phase stacking feature. The atomic model is shown in the center area, and lines 1-6 denote the cationic layer, respectively. b HAADF image extracted from the yellow rectangle in (a), and the intensity profile is calculated from the dark columns (denoted as red rectangle in the lower part) exhibiting a decreasing intensity trend. $\mathbf{c}-\mathbf{h}$ are the atomic mapping resolutions of Ge, Sb, and Te atoms taken from areas 2 and 3, respectively. The EDS profiles along [111] orientation are also presented on the right-hand side of each picture. Scale bar: (a-h) $1 \mathrm{~nm}$

migration behavior in Fig. $2 \mathrm{~g}$, since the peaks of the EDS profiles in lines 2 and 5 are much higher than those in lines 1 and 6 . Meanwhile, the migration of $\mathrm{Ge}$ atoms starts, manifesting as slightly higher peaks in lines 3 and 4 than those of in lines 1, 2, 5, and 6 from its corresponding EDS profile in Fig. 2f. Hence, the migration of $\mathrm{Ge}$ atoms occurs after $\mathrm{Sb}$ atoms. As for Te columns, it always shows a uniform contrast with a nearly same height profile, indicating that $\mathrm{Te}$ atoms as stable as frames here.

To further explore cationic migration in i-phase, GST film was annealed at $290^{\circ} \mathrm{C}$ for $30 \mathrm{~min}$. Figure $3 \mathrm{a}$ is the corresponding HAADF image projected along [1 $1 \overline{1} 0]$ orientation, where lines 1 and 6 show very dark contrasts in HAADF image and the corresponding $\mathrm{Te}-\mathrm{Te}$ atomic spacing above and below the declining cationic layer is about $0.304 \pm 0.007 \mathrm{~nm}$, a value close to the Van der Waals gap spacing expected in h-phase $(0.285 \mathrm{~nm})^{28}$, indicating that more and more $\mathrm{Ge} / \mathrm{Sb}$ atoms have migrated to the neighbor cationic layers. In this i-state, $\mathrm{Ge} / \mathrm{Sb}$ atoms still occupy the cationic sites, and Te atoms occupy anionic sites as shown in Fig. $3 e$ and Supplementary Figure 6. For more, Te always shows a uniform contrast in anionic sites depicted in Fig. 3d, while a vast of Ge and $\mathrm{Sb}$ atoms in lines 1 and 6 have migrated into adjacent nine-layer building blocks as shown in Fig. 3b, c, resulting in lowest peaks in the dark contrast cationic layers. Inspecting the peaks in lines 1 and 6 more carefully, we can find the two peaks are very significant for Ge atoms in Fig. 3b, while no such obvious peaks can be found for $\mathrm{Sb}$ atoms in Fig. 3c, demonstrating a deeper migration behavior for $\mathrm{Sb}$ element. This evidence can also explain the very dark contrast in lines 1 and 6 observed from HAADF image, since the intensity of HAADF is more sensitive for $\mathrm{Sb}(Z=51)$ than the case for $\mathrm{Ge}(Z=$ 32 ), thus a deeper migration behavior of $\mathrm{Sb}$ element would significantly reduce the intensity of HAADF image. Besides, Ge and $\mathrm{Sb}$ elements do not follow the same migration law, and Ge atoms gradually converge to the middle of nine layers building, which is reflected in that lines 3 and 4 have the highest peaks along [111] direction compared to other lines as shown in Fig. $3 \mathrm{~b}$. Sb atoms seem like the edge side of nine layers building block, as shown in
Fig. 3c, and the peaks in lines 2 and 5 are significantly higher than the situation in other lines.

Directly resolving the atomic arrangement of $\mathbf{h}$-phase. Even in the deepest migration case in area 4 , the alloy still retains the cubic stacking sequence. If we keep raising the annealing temperature, cubic lattice of GST alloy will change into h-state. Figure 4 exhibits the atomic EDS mapping results of h-GST

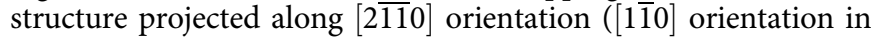
f-phase) after annealing at $350{ }^{\circ} \mathrm{C}$ for $5 \mathrm{~min}$. By inspecting the STEM-HAADF image in Fig. $4 \mathrm{a}$, it is seen that the anionic atoms have a mismatch as denoted by the dashed lines between adjacent building blocks, showing a typical h-phase feature. The distance of Van der Waals gap is about $0.288 \pm 0.008 \mathrm{~nm}$, very close to the value $(0.285 \mathrm{~nm})$ extracted from X-ray diffraction (XRD) experiment $^{28}$. Similar to f-phase, $\mathrm{Ge}$ and $\mathrm{Sb}$ atoms still occupy cationic sites, while Te atoms occupy anionic sites as shown in Supplementary Figure 7. In h-phase, the associated EDS profile along [0001] orientation (corresponding [111] orientation in $\mathrm{f}$ phase) was also inset in the right side of each image, which is integrated in its perpendicular direction, here, the $[0110]$ orientation. From the $\mathrm{Ge} / \mathrm{Sb}$ atomic mapping shown in Fig. 4 b, c, a vast majority of $\mathrm{Ge}$ and $\mathrm{Sb}$ atoms in lines 1 and 6 have already migrated away to other cationic layers. In the blocks, Ge atoms are concentrated in the inner cationic layers (lines 3 and 4), while $\mathrm{Sb}$ atoms like to stay in the outer cationic layers (lines 2 and 5), similar to the situation in area 4 . As for Te element, it still retains a uniform contrast, except a small lattice mismatch between adjacent blocks (denoted by three translucence lines), a fingerprint for h-lattice. Previously, Lotnyk et al. also concluded a similar atomic arrangement by comparing simulation and experimental intensity of the STEM-HAADF image ${ }^{48}$, while in this report we can directly see the atomic arrangement of h-phase of GST alloy from the advanced atomic-resolved EDS mapping results without additional simulation. Furthermore, it is clear that there is a high similarity between $\mathrm{h}$ - and i-phases on the atomic 
a
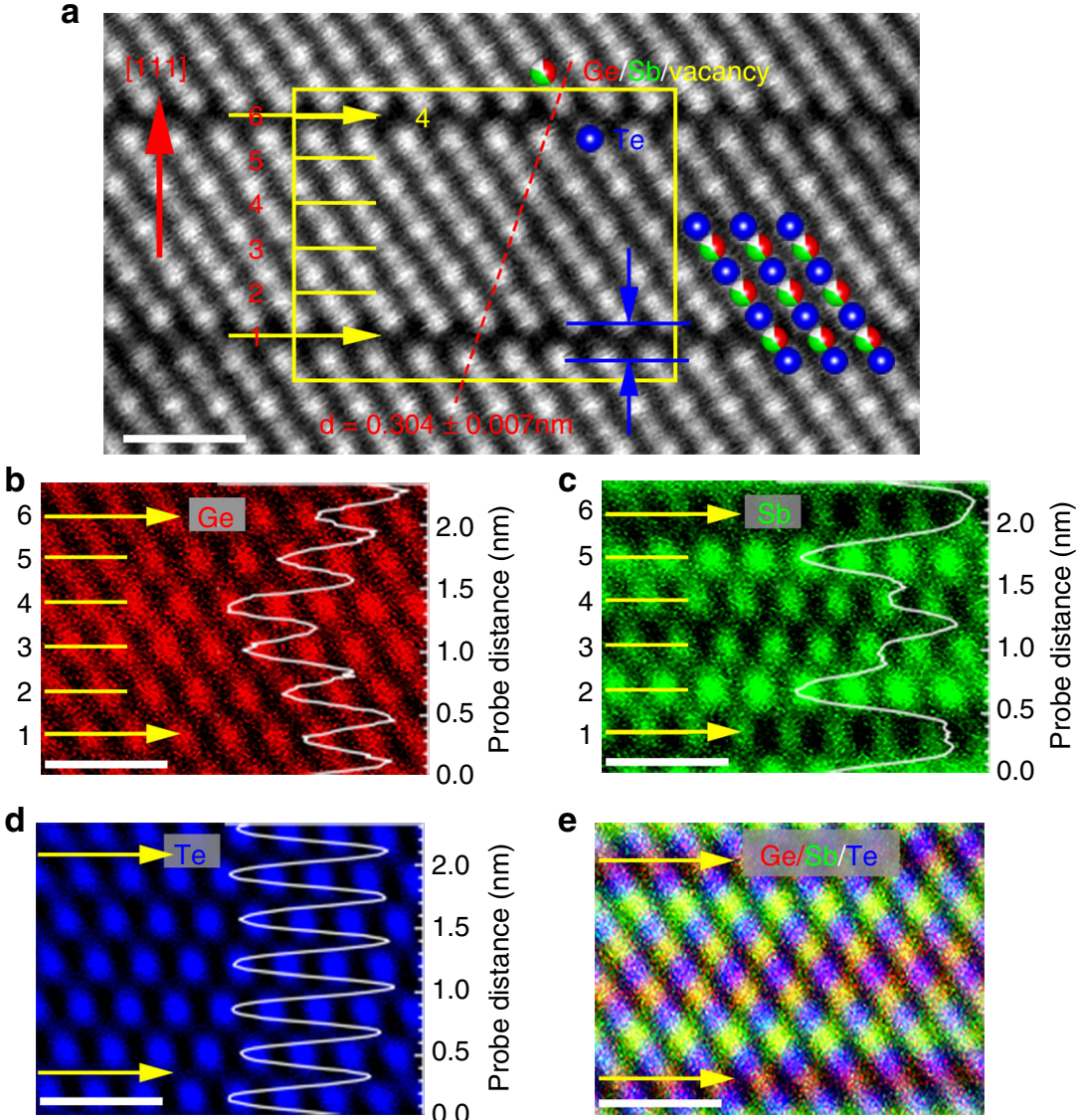

Fig. 3 Atomic resolution elemental maps of i-phase GST at a deeper migration degree. a HAADF image of i-phase GST alloy projected along [110] orientation. Lines 1-6 denote the cationic layer, respectively. b-e The atomic resolution maps of Ge, Sb, and Te atoms and the overlay of three elements taken from area 4 (denoted as yellow rectangle). The EDS profiles along [111] orientation are present on the right-hand side in every picture. Scale bar: (a-e) $1 \mathrm{~nm}$

arrangement side, which strongly suggests that f-to-h structural transition could fluently proceed by gradually cation migration.

Qualitatively, we have effectively traced the migration behavior of $\mathrm{Ge} / \mathrm{Sb}$ elements during f-to-h transition, while it would be much complicated when conducting quantitatively concentration analysis. Roughly, considering that no obvious peaks can be found in lines 1 and 6 , it is reasonable to conclude that Ge and Sb atoms tend to locate in the nine-layer building blocks in h-phase. Therefore, after extracting the EDS counts of each cationic layer for $\mathrm{Ge}$ and $\mathrm{Sb}$ elements from the raw data, we can roughly evaluate the occupancy of $\mathrm{Ge}$ and $\mathrm{Sb}$ atoms in each cationic layer, respectively. Note that, due to the elastic and thermal scattering of the electron probe 49,50 , there is a non-negligible contribution of EDS signal from the nearby columns to the interest columns, and every atomic column (cationic/anionic) has signal of three elements as shown in the Supplementary Note 1, Supplementary Table 1, and Supplementary Figure 8, which would obstruct the actual elements concentration ratio, a similar experiment report was also previously discussed in $\mathrm{SrTiO}_{3}$ sample ${ }^{49}$. Regardless of the intrinsic defects from the electron probe, the occupy ratios of $\mathrm{Ge} / \mathrm{Sb}$ from cationic layer 2 to 5 were plotted (data processing and discussion are shown in the Supplementary Note 2, Supplementary Table 2 and Supplementary Table 3) and compared with the curve calculated from Matsunaga model in Fig. 4e, where the dashed pink represents that the same occupancy ratio for Ge and $\mathrm{Sb}$. The obtained curve for $\mathrm{Ge} / \mathrm{Sb}$ occupancy ratios in each cationic layer still has the same occupancy trend with Matsunaga model, that is, Ge element prefers the inner cationic layers and $\mathrm{Sb}$ element likes the outer cationic layers in the blocks.

Thermally-induced f-to-h phase transition mechanism. After obtaining the atomic distribution in different crystalline states, we can draw a clear scenario of the thermally-induced f-to-h phase transition for GST film at the atomic scale as shown in Fig. 5. At initial f-phase, $\mathrm{Ge}, \mathrm{Sb}$, and vacancies randomly occupy the cationic sites. When the temperature rises, cationic atoms in layer I (corresponding to (111) layer in f-phase) start to migrate to the nearby vacancy sites in layer II, and Sb atoms move earlier than $\mathrm{Ge}$ atoms as denoted by the arrows in Fig. 5a, forming a vacancy aggregation area in layer $\mathrm{I}$ as shown in Fig. 5b afterwards. The earlier $\mathrm{Sb}$ migration behavior may be ascribed to the migration energy barriers difference between Sb and Ge. Previously, Zhang et al. found that the migration energy barrier to move one $\mathrm{Sb}$ atom to the vacancy site is smaller than to move one Ge atom by using the nudged elastic band (NEB) $\operatorname{method}^{25}$. Sun et al. also obtained a similar conclusion from the viewpoint of diffusion energy $^{23}$, specifically, the diffusion energy to move two $\mathrm{Sb}$ atoms from the Te-Te vacancy layer to the $\mathrm{Sb}$ atomic layer $\left(E_{\mathrm{act}}=0.026\right.$ $\mathrm{eV})$ is smaller than the case in $\mathrm{Ge}$ atom $\left(E_{\mathrm{act}}=0.035 \mathrm{eV}\right)$, and the reverse process for both elements would be significantly higher. Afterwards, the rest of cationic atoms in layer I, mainly the Ge atoms, would gradually migrate to layer III via the transition layer II as denoted by the arrows in Fig. 5b. This process also seems to 


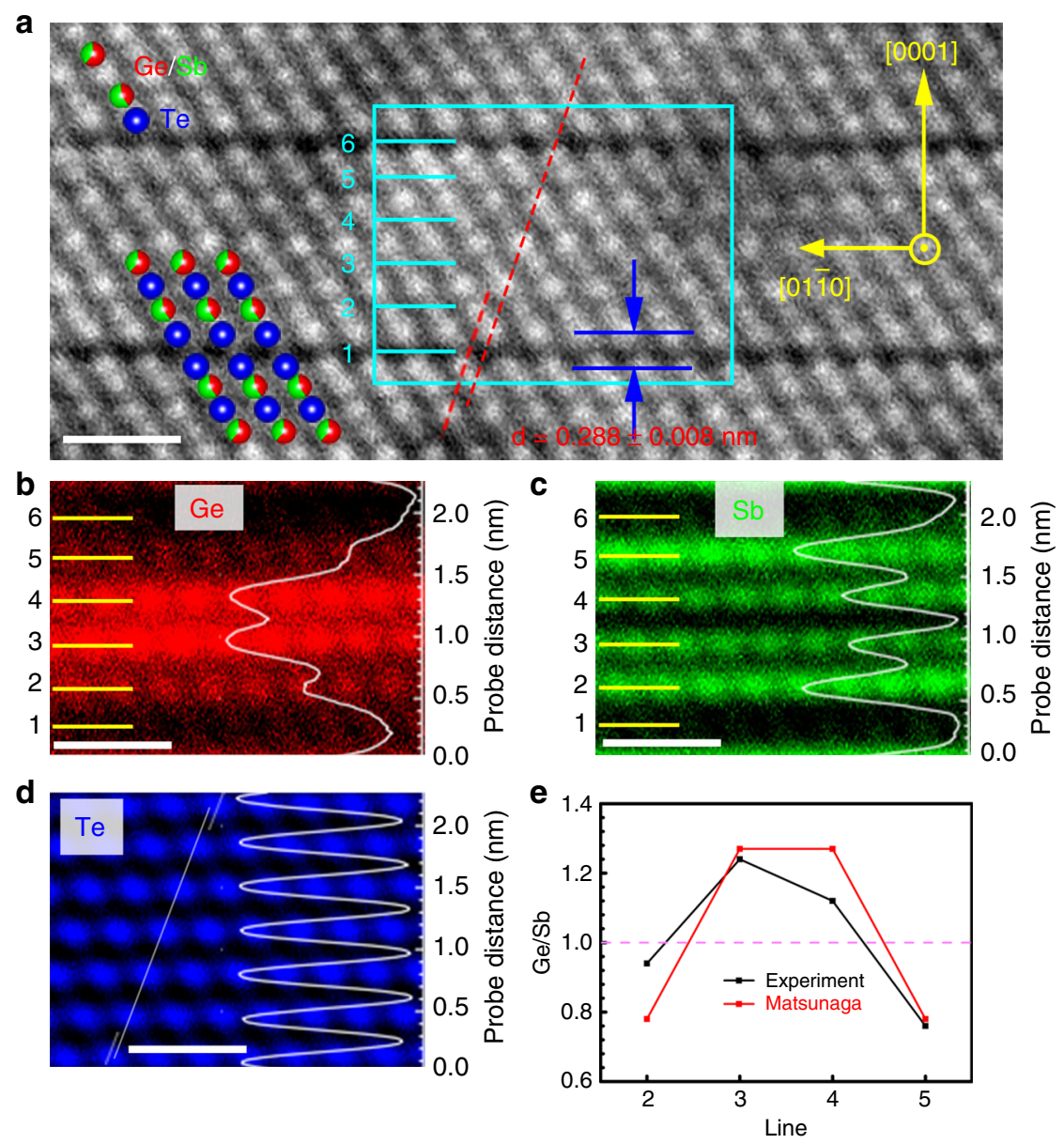

Fig. 4 Atomic resolution elemental maps of h-phase GST. a HAADF image of h-phase GST alloy projected along [2110] orientation. Two dark contrast columns signify the Van der Waals gap labeled as lines 1 and 6, in which the Te-Te distance is about 0.288 $\pm 0.008 \mathrm{~nm}$. Lines 2-5 denote the cationic layer in the nine-layer building block, respectively. b-d The atomic resolution maps pictures of Ge, Sb, and Te atoms taken from cyan rectangle in (a). The EDS profiles along [0001] orientation are present on the right-hand side in every picture. e The Ge/Sb ratio comparison between the experiment result and Matsunaga model. The pink line represents that the occupancy of Ge and Sb is same in cationic layers. Scale bar: (a-d) $1 \mathrm{~nm}$

be related to the energy barrier differences for $\mathrm{Ge}$ and $\mathrm{Sb}$ atoms in layer II after a plenty of $\mathrm{Sb}$ atoms have occupied in layer II, which results in a higher migration energy barrier for Sb atoms. After a majority of $\mathrm{Ge}$ and $\mathrm{Sb}$ atoms in layer I have already migrated to the vacancy sites in layer II and layer III, the bonding between $\mathrm{Te}-\mathrm{Te}$ layers is nearly negligible ${ }^{22}$, and the atomic arrangement in the nine-layer building block of f-phase is very similar to that in the h-structure, ready for the final transformation as shown in Fig. 5c. At last, a slight slip of stacking blocks along [211] orientation ([10 $\overline{1} 0]$ orientation in h-phase) will eventually form the h-structure in Fig. 5d, with a stacking sequence similar to Matsunaga model.

Though many literatures claim that Kooi sequence is the stable configuration in h-phase, in which Sb completely occupy the outer layers and Ge occupy the inner layers, the hierarchy in energy between Kooi sequence and Matsunaga sequence is very small (just less than $5 \mathrm{meV}$ per atom), indicating that the mentioned stacking sequences are both energy favorable. In fact, the difference in energy between Kooi sequence and Matsunaga sequence reported by Sun et al. ( $2 \mathrm{meV} / \mathrm{atom})$ is of the same order of magnitude expected for the free energy contribution (at $300 \mathrm{~K})$ due to the configuration entropy of the disordered (intermixing) Matsunaga sequence $\left(\frac{4}{9} k_{B} T \operatorname{In} 2=8 \mathrm{meV} /\right.$ atom, in which $k_{B}$ is the Boltzmann constant, $T$ is the absolute temperature) as pointed by Caravati et al. ${ }^{34}$. One may doubt the possible atomic movement process making Matsunaga sequence transform into Kooi sequence induced by further annealing, for example, interchanging the position between $\mathrm{Ge}$ and $\mathrm{Sb}$ atoms in the blocks. After the cationic migration process, the majority of the vacant positions would be occupied by Ge or $\mathrm{Sb}$ atoms except for Van der Waals gap, though there may be still some excess vacancies exist in the nine-layer building according to the conducting transport measurements ${ }^{51}$, such concentrations of excess vacancies compatible with the experimental carrier densities are very small. Thus, the proposed interchanging process seems to be difficult than the migration behavior which was assisted with a lot of random vacancies in f-phase. Interestingly, Matsunaga even found that the mixture $\mathrm{Ge} / \mathrm{Sb}$ situation in h-phase still retains when annealed up to $923 \mathrm{~K}^{28}$, close to the melting temperature of the alloy ${ }^{52}$. Therefore, we speculate that the obtained Matsunaga sequence-like h-phase arrangement is a stable configuration which is much related to the forming process, that is, the different migration behaviors of $\mathrm{Ge}$ and $\mathrm{Sb}$ atoms makes an unbalanced cationic distribution in the obtained h-phase. Besides, the present configuration also leads to a more favorable charge balance in the three coordinated edge $\mathrm{Te}$ atoms, since $\mathrm{Sb}^{\mathrm{III}}$ atoms possess a higher oxidation state than $\mathrm{Ge}$ [II 53 . 
a

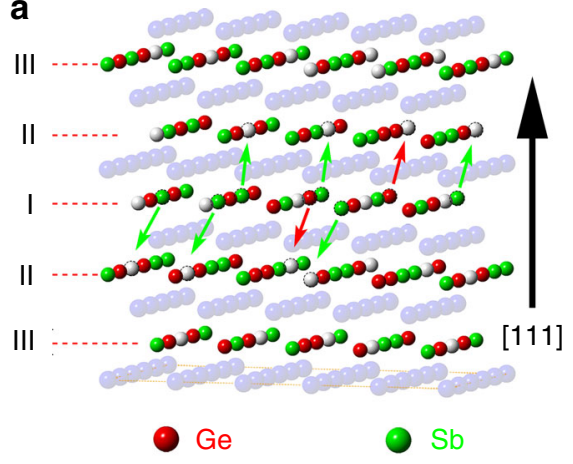

C

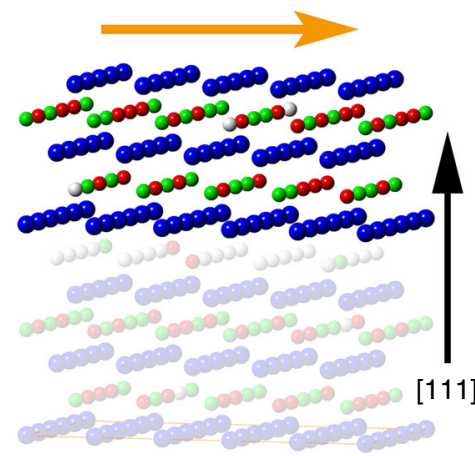

b

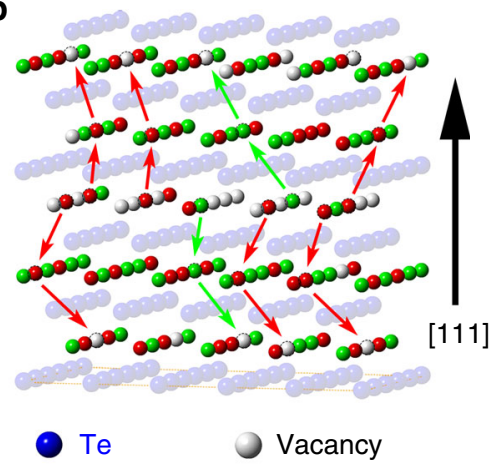

d

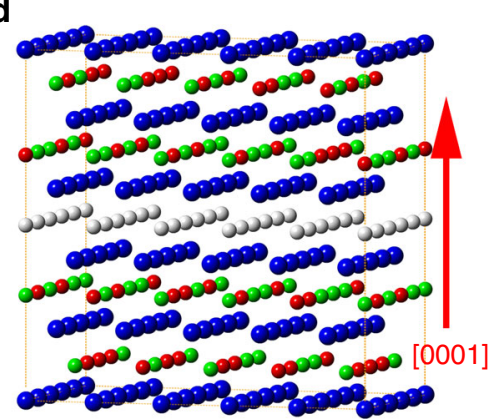

Fig. 5 A schematic scenario of the f-to-h phase transition. a The initial f-phase projected along [111] direction, in which cationic sites are randomly occupied by $\mathrm{Ge}, \mathrm{Sb}$, and vacancy. Red and green arrows denote the possible migration paths of the cationic atoms from layer I to layer II induced by thermal annealing. $\mathbf{b}$ The atomic configuration of i-phase after the migration process described in (a). Red and green arrows denote the possible migration paths of the cationic atoms from layer I to layer III via the transition layer II afterwards. c The atomic configuration of a deeper migration extent i-phase after the majority of $\mathrm{Ge}$ and $\mathrm{Sb}$ atoms in layer I have migrated, h-lattice-like atom arrangement makes the stacking block easily to slip to transition into h-phase. Orange arrow denotes the slip direction of the block. $\mathbf{d}$ The atomic configuration for the stable h-phase projected along [0001] direction, where Ge element prefers the inner cationic layers and Sb element likes the outer cationic layers in the blocks

\section{Discussion}

In summary, by utilizing advanced atomic-resolved EDS mapping technique, we systematically explore the atomic arrangement of different crystalline state during f-to-h phase transformation. Under external thermal annealing, randomly distributed Ge and $\mathrm{Sb}$ atoms gradually migrate to the neighbor (111) cationic layers, forming nine-layer blocks of i-phase very similar to h-lattice. The migration process is partial orderly, $\mathrm{Sb}$ atoms migrate earlier than $\mathrm{Ge}$ atoms, meanwhile, Ge element prefers the inner cationic layers, and $\mathrm{Sb}$ element likes the outer cationic layers in the formed h-lattice-like stacking blocks. The deeper Ge/Sb migration degree of $\mathrm{i}$-phase, the closer the atomic arrangement is to the hlattice. In the final nine-layer building block of h-phase, $\mathrm{Sb}$ atoms aggregate in the outer cationic layers, and Ge atoms aggregate in the inner cation layers, forming the following suggested stacking sequence: $\quad-\mathrm{Te}-\mathrm{Sb}_{x} / \mathrm{Ge}_{y}-\mathrm{Te}-\mathrm{Ge}_{x} / \mathrm{Sb}_{y}-\mathrm{Te}-\mathrm{Ge}_{x} / \mathrm{Sb}_{y}-\mathrm{Te}-\mathrm{Sb}_{x} / \mathrm{Ge}_{y^{-}}$ $\mathrm{Te}-(x>y)$ which is similar to the Matsunaga model. In short, through the visual atomic identification of $\mathrm{f}$-to-h transition fragments in GST material, we have connected the whole transition process at atomic level step by step, and successfully solved a crucial problem which has been a source of controversy and debate in the past. At the atomic scale, the key to prevent the formation of h-phase in GST should restrain the migration behavior of $\mathrm{Ge}$ and $\mathrm{Sb}$ atoms, for example, doping appropriate element into the cationic sites to reduce the vacancy concentration. Undoubtedly, the current results should act as a solid experiment basis for understanding the microscopic properties in GST alloy and pave the way of further application of phase change memory. Further work is also needed to clarify the atomic arrangement in $\mathrm{GeTe}-\mathrm{Sb}_{2} \mathrm{Te}_{3}$ pseudobinary alloy and the origin of the migration behavior in the phase change material.

\section{Methods}

Sample preparation. GST films ( $\sim 500 \mathrm{~nm}$ in thickness) were directly deposited on silicon (100) substrate at room temperature by sputtering a single GST alloy target. The base pressure of the vacuum system is about $2.1 \times 10^{-4} \mathrm{~Pa}$, the Ar flow rate is $80 \mathrm{sccm}$ and the corresponding sputtering pressure is about $0.31 \mathrm{~Pa}$. To obtain a series of crystalline structures, the films undergone thermal annealing process from 150 to $350^{\circ} \mathrm{C}$ for different times in rapid thermal processing in an argon atmosphere. By using focus ion beam (FIB) technique, the annealed films were fabricated into cross-section samples in FEI Helios 600 instrument with standard lift out and polishing process, and then cleaned in Gatan 691 PIPS at $0.8 \mathrm{keV}$ for removing the residual contamination and possible damage.

Microstructure characterization. The STEM-HAADF images were captured using the STEM mode of JEM Grand ARM300F microscope with double spherical aberration (Cs) correctors, in which, the inner semi-angle for the dark field detector is larger than $63 \mathrm{mrad}$. Two windowless EDS detectors, each of which has an active area of $100 \mathrm{~mm}^{2}$, are equipped on JEM Grand ARM300F microscope, which are very close to the specimen with a high solid angle $(1.7 \mathrm{sr})$. By using of the EDS detectors, the average concentrations of Ge, Sb, and Te elements of the GST films were determined to be $19.1,24.5$, and 56.4 at \%, i.e., a value close to $\mathrm{Ge}_{2} \mathrm{Sb}_{2} \mathrm{Te}_{5}$. Since chalcogenide alloy is very sensitive to the accelerate electron beam, the operating voltage is under $80 \mathrm{kV}$ to reduce the damage when conducting atomic EDS experiment and obtaining HAADF images, and in turn has a relatively not good enough signal-noise ratio in the results. Comparing the HAADF image before and after electron beam irradiation, no obvious artificial has been found except for a slight electron damage as shown in Supplementary Figure 1. A convergence semi-angle of $24 \mathrm{mrad}$ and inner acquisition semi-angle of $63 \mathrm{mrad}$ were used in STEM mode. The electron beam current is about $30 \mathrm{pA}$. The STEM probe size is around $0.08 \mathrm{~nm}$ which corresponds to the specified spatial resolution of the STEM instrument. All the raw data of EDS were processed with Wiener filtering which has been widely used to remove noise in HAADF images $45,54,55$. It has 
shown that no artefacts are found when comparing Wiener filtering images with raw images ${ }^{56}$ which was also confirmed in Supplementary Figure 2, implying that we can use Wiener filtering images to effectively identify the location of atomic columns, and qualitatively analyze the corresponding concentration variation in a small area, for example, the interface or the surface area of the nanostructure. Supplementary Figure 3 is a typical atomic EDS mapping result for $\mathrm{SrTiO}_{3}$ sample fitting well with the known cubic perovskite structure.

\section{Data availability}

The data that support the findings of this study are available within the article and its Supplementary Information files. All other relevant source data are available from the corresponding author upon reasonable request.

Received: 24 August 2018 Accepted: 7 January 2019

Published online: 01 February 2019

\section{References}

1. Salinga, M. \& Wuttig, M. Phase-change memories on a diet. Science 332, 543-544 (2011).

2. Wong, H.-S. P. \& Salahuddin, S. Memory leads the way to better computing. Nat. Nanotechnol. 10, 191-194 (2015).

3. Rao, F. et al. Reducing the stochasticity of crystal nucleation to enable subnanosecond memory writing. Science 358, 1423-1427 (2017).

4. Wuttig, M. \& Yamada, N. Phase-change materials for rewriteable data storage. Nat. Mater. 6, 824-832 (2007).

5. Yamada, N. et al. Rapid-phase transitions of $\mathrm{GeTe}-\mathrm{Sb}_{2} \mathrm{Te}_{3}$ pseudobinary amorphous thin films for an optical disk memory. J. Appl. Phys. 69, 2849 (1991).

6. Kim, W. et al. ALD-based confined PCM with a metallic liner toward unlimited endurance. In Proceedings of the 2016 IEEE International Electron Devices Meeting (IEDM) 4.2.1-4.2.4 (2016).

7. Im, D. H. et al. A unified $7.5 \mathrm{~nm}$ dash-type confined cell for high performance PRAM device. In Proceedings of the 2008 IEEE International Electron Devices Meeting (IEDM) 1-4 (2008).

8. Wang, Y. et al. Scandium doped $\mathrm{Ge}_{2} \mathrm{Sb}_{2} \mathrm{Te}_{5}$ for high-speed and low-powerconsumption phase change memory. Appl. Phys. Lett. 112, 133104 (2018).

9. Zhou, X. et al. Carbon-doped $\mathrm{Ge}_{2} \mathrm{Sb}_{2} \mathrm{Te}_{5}$ phase change material: a candidate for high-density phase change memory application. Appl. Phys. Lett. 101, 142104 (2012).

10. Lankhorst, M. H. R. et al. Low-cost and nanoscale non-volatile memory concept for future silicon chips. Nat. Mater. 4, 347-352 (2005).

11. Wang, Y. et al. Understanding the early cycling evolution behaviors for phase change memory application. J. Appl. Phys. 116, 204503 (2014).

12. Hegedüs, J. \& Elliott, S. R. Microscopic origin of the fast crystallization ability of Ge-Sb-Te phase-change memory materials. Nat. Mater. 7, 399-405 (2008).

13. $\mathrm{Li}, \mathrm{T}$. et al. Carbon doping induced $\mathrm{Ge}$ local structure change in as-deposited $\mathrm{Ge}_{2} \mathrm{Sb}_{2} \mathrm{Te}_{5}$ film by EXAFS and Raman spectrum. AIP Adv. 8, 025201 (2018).

14. Wang, Y. et al. RESET distribution improvement of phase change memory: the impact of Pre-programming. IEEE Electron Device Lett. 35, 536-538 (2014).

15. Njoroge, W. K., Wöltgens, H.-W. \& Wuttig, M. Density changes upon crystallization of $\mathrm{Ge}_{2} \mathrm{Sb}_{2.04} \mathrm{Te}_{4.74}$ films. J. Vac. Sci. Technol. 20, 230-233 (2001).

16. Cheng, H. Y. et al. A high performance phase change memory with fast switching speed and high temperature retention by engineering the $\mathrm{Ge}_{x} \mathrm{Sb}_{y} \mathrm{Te}_{z}$ phase change material. In Proceedings of the 2011 IEEE International Electron Devices Meeting (IEDM) 3.4.1-3.4.4 (2011).

17. Park, I. M. et al. Thermomechnaical properties and mechanical stresses of $\mathrm{Ge}_{2} \mathrm{Sb}_{2} \mathrm{Te}_{5}$ films in phase-change random access memory. Thin Solid Films 517, 848-852 (2008).

18. Do, K. et al. TEM study on volume changes and void formation in GeSbTe fims, with repeated phase changes. Electrochem. Solid-State Lett. 13, H284-H286 (2010).

19. Simpson, R. E. et al. Interfacial phase-change memory. Nat. Nanotechnol. 6, 501-505 (2011).

20. Yamada, N. \& Matsunaga, T. Structure of laser-crystallized $\mathrm{Ge}_{2} \mathrm{Sb}_{2+x} \mathrm{Te}_{5}$ sputtered thin films for use in optical memory. J. Appl. Phys. 88, 7020 (2000).

21. Eom, J.-H. et al. Global and local structures of the Ge-Sb-Te ternary alloy system for a phase-change memory device. Phys. Rev. B 73, 214202 (2006).

22. Sun, Z., Zhou, J. \& Ahuja, R. Structure of phase change materials for data storage. Phys. Rev. Lett. 96, 055507 (2006).

23. $\mathrm{He}$, S. et al. Metastable stacking-polymorphism in $\mathrm{Ge}_{2} \mathrm{Sb}_{2} \mathrm{Te}_{5}$. Inorg. Chem. 56, 11990-11997 (2017)
24. Zhang, B. et al. Vacancy structures and melting behavior in rock-salt GeSbTe. Sci. Rep. 6, 25453 (2016).

25. Zhang, B. et al. Element-resolved atomic structure imaging of rocksalt $\mathrm{Ge}_{2} \mathrm{Sb}_{2} \mathrm{Te}_{5}$ phase-change material. Appl. Phys. Lett. 108, 191902 (2016).

26. Kooi, B. J., Groot, W. M. G. \& Hosson, J. T. M. D. In situ transmission electron microscopy study of the crystallization of $\mathrm{Ge}_{2} \mathrm{Sb}_{2} \mathrm{Te}_{5}$. J. Appl. Phys. 95, 924 (2004).

27. Petrov, I. I., Imamov, R. M. \& Pinsker, Z. G. Electron-diffraction determination of the structures of $\mathrm{Ge}_{2} \mathrm{Sb}_{2} \mathrm{Te}_{5}$ and $\mathrm{GeSb}_{4} \mathrm{Te}_{7}$. Sov. Phys. Crystallogr. 13, 339-342 (1968).

28. Matsunaga, T., Yamada, N. \& Kubota, Y. Structures of stable and metastable $\mathrm{GeSbTe}$, an intermetallic compound in $\mathrm{GeTe}-\mathrm{S}_{\mathrm{b}} 2 \mathrm{~T}_{\mathrm{e}} 3$ pseudobinary systems. Acta Crystallogr. Sect. B 60, 685-691 (2004).

29. Yu, X. \& Robertson, J. Atomic layering, intermixing and switching mechanism in Ge-Sb-Te based chalcogenide superlattices. Sci. Rep. 6, 37325 (2016).

30. Hernández, W. I. \& Raty, J.-Y. Ab initio density functional theory study of the electronic, dynamic, and thermoelectric properties of the crystalline pseudobinary chalcogenide $(\mathrm{GeTe}) x /\left(\mathrm{Sb}_{2} \mathrm{Te}_{3}\right)(x=1,2,3)$. Phys. Rev. B 97, 245205 (2018)

31. Singh, J. et al. Effect of gradual ordering of $\mathrm{Ge} / \mathrm{Sb}$ atoms on chemical bonding a proposed mechanism for the formation of crystalline $\mathrm{Ge}_{2} \mathrm{Sb}_{2} \mathrm{Te}_{5}$. J. Solid State Chem. 260, 124-131 (2018).

32. Da Silva, J. L. F., Walsh, A. \& Lee, H. Insights into the structure of the stable and metastable $(\mathrm{GeTe})_{m}\left(\mathrm{Sb}_{2} \mathrm{Te}_{3}\right)_{n}$ compunds. Phys. Rev. B 78, 22411 (2008).

33. Lee, G. \& Jhi, S.-H. Ab initio studies of structural and electronic properties of the crystalline $\mathrm{Ge}_{2} \mathrm{Sb}_{2} \mathrm{Te}_{5}$. Phys. Rev. B 77, 153201 (2008).

34. Sosso, G. C. et al. Vibrational properties of hexagonal $\mathrm{Ge}_{2} \mathrm{Sb}_{2} \mathrm{Te}_{5}$ from first principles. J. Phys. Condens. Matter 21, 245401 (2009).

35. Lotnyk, A. et al. Local atomic arrangements and lattice distortions in layered Ge-Sb-Te crystal structures. Sci. Rep. 6, 26724 (2016).

36. Mio, A. M. et al. Chemical and structural arrangement of the trigonal phase in GeSbTe thin films. Nanotechnology 28, 065706 (2017).

37. Lotnyk, A. et al. Real-space imaging of atomic arrangement and vacancy layer ordering in laser crystallised GeSbTe phase change thin films. Acta Mater. 105, 1-8 (2016)

38. Zhang, W., Jeong, H. S. \& Song, S. A. Martensitic transformation in $\mathrm{Ge}_{2} \mathrm{Sb}_{2} \mathrm{Te}_{5}$ alloy. Adv. Eng. Mater. 10, 67-72 (2008).

39. Zhang, W. et al. How important is the $\{103\}$ plane of stable GeSbTe for phase change memory? J. Microsc. 259, 10-15 (2015).

40. Park, Y. J., Lee, J. Y. \& Kim, Y. T. In situ transmission electron microscopy study of the nucleation and grain growth of $\mathrm{Ge}_{2} \mathrm{Sb}_{2} \mathrm{Te}_{5}$ thin films. Appl. Surf. Sci. 252, 8102-8106 (2006).

41. Kim, E. T., Lee, J. Y. \& Kim, Y. T. Investigation of the structural transformation behavior of $\mathrm{Ge}_{2} \mathrm{Sb}_{2} \mathrm{Te}_{5}$ thin films using high resolution electron microscopy. Appl. Phys. Lett. 91, 101909 (2007)

42. Zhang, W. et al. Role of vacancies in metal-insulator transitions of crystalline phase-change material. Nat. Mater. 11, 952-956 (2012).

43. Bragaglia, V. et al. Metal-insulator transition driven by vacancy ordering in GeSbTe phase change materials. Sci. Rep. 6, 23843 (2016).

44. Williams, D. B. \& Carter, C. B. Transmission Electron Microscopy: A Textbook for Materials Science. 2nd ed., (Springer, Berlin, 2009).

45. Ross, U. et al. Direct imaging of crystal structure and defects in metastable GeSbTe by quantitative aberration-corrected scanning transmission electron microscopy. Appl. Phys. Lett. 104, 121904 (2014).

46. Jiang, Y. et al. Direct atom-by-atom chemical identification of nanostructures and defects of topological insulators. Nano Lett. 13, 2851-2856 (2013).

47. Rao, F. et al. Direct observation of titanium-centered octahedral in titanium-antimony-tellurium phase-change material. Nat. Commun. 6, 10040 (2015).

48. Lotnyk, A. et al. Van der Waals interfacial bonding and intermixing in $\mathrm{GeTe}-\mathrm{Sb}_{2} \mathrm{Te}_{3}$-based superlattices. Nano Res. 11, 1676-1686 (2018).

49. Kothleiner, G. et al. Quantitative elemental mapping at atomic resolution using X-ray spectroscopy. Phys. Rev. Lett. 112, 085501 (2014).

50. Lugg, N. R. et al. On the quantitativeness of EDS STEM. Ultramicroscopy 151 , 150-159 (2015).

51. Bong-Sub, L. et al. Investigation of the optical and electronic properties of $\mathrm{Ge}_{2} \mathrm{Sb}_{2} \mathrm{Te}_{5}$ phase change material in its amorphous, cubic and hexagonal phases. J. Appl. Phys. 97, 093509 (2005).

52. Kalb, J., Spaepen, F. \& Wuttig, M. Calorimetric measurements of phase transformations in thin films of amorphous Te alloys used for optical data storage. J. Appl. Phys. 93, 2389 (2003).

53. Urban, P. et al. Temperature dependent resonant X-ray diffraction of singlecrystalline $\mathrm{Ge}_{2} \mathrm{Sb}_{2} \mathrm{Te}_{5}$. CrystEngComm 15, 4823-4829 (2013).

54. Chen, C.-C. et al. Three-dimensional imaging of dislocations in a nanoparticle at atomic resolution. Nature 496, 74-77 (2013).

55. Xu, R. et al. Three-dimensional coordinates of individual atoms in materials revealed by electron tomography. Nat. Mater. 14, 1099-1103 (2015). 
56. Li, J. et al. Surface evolution of a Pt-Pd-Au electrocatalyst for stable oxygen reduction. Nat. Energy 2, 17111 (2017)

\section{Acknowledgements}

This work is supported by the National Key Research and Development Program of China (2017YFA0206101), "Strategic Priority Research Program" of the Chinese Academy of Sciences (XDA09020402), and National Integrate Circuit Research Program of China (2009ZX02023-003).

\section{Author contributions}

Y.Z. and Y.W. prepared the film samples. S.L. and Y.C. conducted the FIB experiments. Y.Z., T.X., and Y.C. carried out the transmission electron microscopy experiments. Analysis of the data was mostly carried out by Y.Z., Y.W., and Y.C. with valuable suggestions from R.H., P.L., M.L., and Z.Z. Y.Z. and Y.C. wrote the paper with contributions from all co-authors. The project was initiated and conceptualized by Z.S. and S.F.

\section{Additional information}

Supplementary information accompanies this paper at https://doi.org/10.1038/s42004019-0114-7.

Competing interests: The authors declare no competing interests.
Reprints and permission information is available online at http://npg.nature.com/ reprintsandpermissions/

Publisher's note: Springer Nature remains neutral with regard to jurisdictional claims in published maps and institutional affiliations.

\section{(c) (1)}

Open Access This article is licensed under a Creative Commons Attribution 4.0 International License, which permits use, sharing, adaptation, distribution and reproduction in any medium or format, as long as you give appropriate credit to the original author(s) and the source, provide a link to the Creative Commons license, and indicate if changes were made. The images or other third party material in this article are included in the article's Creative Commons license, unless indicated otherwise in a credit line to the material. If material is not included in the article's Creative Commons license and your intended use is not permitted by statutory regulation or exceeds the permitted use, you will need to obtain permission directly from the copyright holder. To view a copy of this license, visit http://creativecommons.org/ licenses/by/4.0/.

(C) The Author(s) 2019 